\title{
The Classical Problem of Water Waves: a Reservoir of Integrable and Nearly-Integrable Equations
}

\author{
Robin S JOHNSON
}

School of Mathematics 8 Statistics, University of Newcastle upon Tyne, Newcastle upon Tyne, NE1 $7 R U, U K$

E-mail:R.S.Johnson@ncl.ac.uk

This paper is part of the

Proceedings of the Öresund Symposium on Partial Differential Equations;

Lund University, May 23-25, 2002

\begin{abstract}
In this contribution, we describe the simplest, classical problem in water waves, and use this as a vehicle to outline the techniques that we adopt to analyse this particular approach to the derivation of soliton-type equations. The surprise, perhaps, is that such an apparently transparent set of equations (the Euler equation for an incompressible fluid, the equation of mass conservation and the simplest bottom and surface conditions) contains so may different - and important - equations.

We will briefly show how such equations are generated, by carefully describing the asymptotic procedures that we adopt. We will then present a number of examples, some of which will be the familiar integrable equations (Korteweg-de Vries (KdV), Nonlinear Schrödinger (NLS)) and also variants of these which are, in a sense, nearlyintegrable. These will include, for example, a variable coefficient $\mathrm{KdV}$ equation, a nearly concentric KdV equation and higher-order NLS equation. Some of these results have significant consequences for water-wave propagation; for example, the existence of a KdV equation for arbitrary velocity profiles below the surface helps to explain why solitary waves are observed in nature.

We conclude with a discussion of some of the more recent, exciting work on the rôle of the Camassa-Holm $(\mathrm{CH})$ equation in water waves. We will also outline how, for this type of problem, a number of interesting and relevant variants of the $\mathrm{CH}$ equation can arise.
\end{abstract}

\section{Introduction}

This contribution to the symposium is intended to give an overview of the many and varied integrable and nearly-integrable - we will explain what we mean by this terminology later - equations that can be extracted from the simplest, classical problem in water waves. This will highlight the rôle of these important equations within the framework of a physical problem, even though we will describe a model which views the fluid and the motion in a rather simplistic way. Nevertheless, we are able to give an interpretation of 
these equations within a specific context and, more significantly (we believe), show how such equations are altered and extended when further embellishments are included in the modelling process. There are various general aspects that we will touch upon, including: the assumptions that underpin the existence of these equations (albeit in an asymptotic sense); the relations, if any, which exist between different approximations of the waterwave problem; the way in which these equations, and their solutions, contribute to the complete description of the propagation processes (which typically comprises a number of wave components).

The idealised flow that we analyse is that of an inviscid fluid, although the flow field may be rotational. Further, the surface of the water will be assumed to exhibit only those characteristics which are associated with zero surface tension. Consequently, therefore, our model will exclude all turbulent features, any wind/wave interaction (other than that generated by the normal stress : constant pressure at the surface) and will describe only gravity waves - capillary waves cannot appear. Nevertheless, we can still produce interesting and useful models that can include contributions from, for example, variable depth and general, prescribed motion of the water (usually called a 'shear' flow). The evidence of the resulting analyses suggests that much of the work is broadly relevant to water waves that are observed in nature, if suitable averaging techniques are used to interpret the turbulent properties of the real flow, the long-term effects of viscosity ignored, et cetera. However, the main intellectual challenge is to understand what the equations have to tell us about these flow problems, and about nonlinear wave propagation more generally. Such studies have a long and exalted history; see, for example, [16, 39, 40, 4, 38, 32, 17]. Indeed, these investigations provide an excellent example of the approach adopted in modern applied mathematics: start from a suitable set of governing equations; introduction of nondimensionalisation and scaling; rational approximation; solution of equations; interpretation.

First, we formulate the classical water-wave problem, introduce an appropriate set of nondimensional variables and then scale; this leads to a set of equations (and boundary conditions) which contain two fundamental parameters. The sizes, and relative size, of these parameters then govern the type of problem under consideration; this, in turn, stipulates the nature of the initial data which would lead to the evolution of the solution that follows from the particular choice of parameters. We shall briefly describe the familiar development that leads to the Korteweg-de Vries (KdV) and Nonlinear Schrödinger (NLS) equations - two of the archetypal equations of soliton theory. (An introduction to the ideas of soliton theory and, in particular, their application to the KdV and NLS equations, can be found in [12].) Interestingly, within the context of our problem, these two equations are directly related: they match, in an appropriate asymptotic sense. Then we extend these derivations by generalising the problem under consideration, by working in different geometries, by including variable depth and by adding an underlying shear flow. (The use of the word 'shear' is not to imply a viscous phenomenon; the ambient flow, in the absence of any waves, simply possesses non-zero vorticity. This model allows us to represent a more physically realistic flow in which a velocity profile exists, a profile which may, in the original physical problem, be controlled by viscous stresses.) All these examples give rise to variants of the two pre-eminent, classical equations; we conclude with a discussion of the rôle of the Camassa-Holm equation in water waves - an equation which has caused some excitement in recent years. 


\section{Governing equations}

We consider an incompressible, inviscid fluid, with zero surface tension, moving over an impermeable bed; at the surface, we impose the simple condition of constant pressure $\left(=P_{a}\right.$, atmospheric pressure). Thus the governing equations are

$$
\frac{D \boldsymbol{u}}{D t}=-\frac{1}{\rho} \nabla P+\boldsymbol{F}, \quad \nabla \cdot \boldsymbol{u}=0,
$$

with

$$
P=P_{a} \quad \text { and } \quad w=\frac{D h}{D t} \quad \text { both on } \quad z=h\left(\boldsymbol{x}_{\perp}, t\right)
$$

and

$$
w=\left(\boldsymbol{u}_{\perp} \cdot \nabla_{\perp}\right) b \quad \text { on } \quad z=b\left(\boldsymbol{x}_{\perp}\right),
$$

where $\boldsymbol{F}=(0,0,-g), g$ constant, $\rho$ is the (constant) density of the water, $\boldsymbol{u}=\left(\boldsymbol{u}_{\perp}, w\right)$ is the velocity in the flow and $D / D t$ is the usual material derivative. The surface is represented by $z=h\left(\boldsymbol{x}_{\perp}, t\right)$, where $\boldsymbol{x}_{\perp}$ denotes the 2 -vector which is perpendicular to the direction of the $z$-coordinate (which is upwards); the impermeable bed $\left(z=b\left(\boldsymbol{x}_{\perp}\right)\right.$ ) is taken to be steady. (A prescribed dependence on time, $t$, could be used to model, for example, submarine quakes.) The usual aim in these studies is to find (approximate) solutions for $h\left(\boldsymbol{x}_{\perp}, t\right)$, although the underlying flow may also be of some interest; we are given a suitable ambient flow environment in which the waves propagate together with appropriate initial data.

The first stage in any analysis of this type is to nondimensionalise these equations, (2.1)(2.3), by using some suitable general scales that define the class of problems that we wish to address. Let $h_{0}$ be a typical undisturbed depth of the water (perhaps an average depth or the constant depth over which the wave initially moves, before entering a region of variable depth) and let $\lambda$ be a typical wavelength of the surface wave. A relevant speed scale is then $\sqrt{g h_{0}}$ and so a corresponding time scale associated with the passage of the wave is $\lambda / \sqrt{g h_{0}}$. The speed scale $\sqrt{g h_{0}}$ is used only for the horizontal velocity components $\left(\boldsymbol{u}_{\perp} \equiv(u, v)\right)$; to be consistent with the equation of mass conservation and the surface kinematic condition, the vertical component, $w$, of the velocity vector is nondimensionalised by $h_{0} \sqrt{g h_{0}} / \lambda$. Thus we nondimensionalise according to

$$
\begin{aligned}
& \boldsymbol{x}_{\perp} \rightarrow \lambda \boldsymbol{x}_{\perp}, \quad z \rightarrow h_{0} z, \quad t \rightarrow\left(\lambda / \sqrt{g h_{0}}\right) t \\
& \boldsymbol{u}_{\perp} \rightarrow \sqrt{g h_{0}} \boldsymbol{u}_{\perp}, \quad w \rightarrow\left(h_{0} \sqrt{g h_{0}} / \lambda\right) w
\end{aligned}
$$

where the arrow is to be read as 'replace by', so that all the same notation is retained, but hereafter all these symbols denote nondimensional variables. Further, we write

$$
h=h_{0}+a \eta \quad \text { and } \quad b \rightarrow h_{0} b,
$$

where $a$ is a measure of wave amplitude, and define the pressure as

$$
P=P_{a}+\rho g\left(h_{0}-z\right)+\rho g h_{0} p
$$


where this $z$ is still dimensional, but $p$ is now the nondimensional pressure which measures the deviation away from the hydrostatic pressure distribution. Using equations (2.4)-(2.6) in (2.1)-(2.3) gives us the set

$$
\frac{D}{D t} \boldsymbol{u}_{\perp}=-\nabla_{\perp} p, \quad \delta^{2} \frac{D w}{D t}=-\frac{\partial p}{\partial z}, \quad \nabla \cdot \boldsymbol{u}=0,
$$

with

$$
p=\varepsilon \eta \quad \text { and } \quad w=\varepsilon\left\{\frac{\partial \eta}{\partial t}+\left(\boldsymbol{u}_{\perp} \cdot \nabla_{\perp}\right) \eta\right\} \quad \text { on } \quad z=1+\varepsilon \eta\left(\boldsymbol{x}_{\perp}, t\right)
$$

and

$$
w=\left(\boldsymbol{u}_{\perp} \cdot \nabla_{\perp}\right) b \quad \text { on } \quad z=b\left(\boldsymbol{x}_{\perp}\right) .
$$

Here, we have introduced the two fundamental parameters that appear in the problem: $\varepsilon=a / h_{0}$, the amplitude parameter, and $\delta=h_{0} / \lambda$, the long wavelength (or shallowness) parameter; we shall write much of their rôles shortly. The final stage, leading to the equations with which we shall work, is to scale with respect to $\varepsilon$.

The case $\varepsilon=0$ corresponds to undisturbed conditions i.e. no wave is present; accordingly, as $\varepsilon \rightarrow 0$, so the disturbances associated with the passage of the wave vanish. Although we may not always wish to take this limit - we could elect to study the 'fully' nonlinear problem described by $\varepsilon=1$ - the equations must be consistent with this choice. This requires that we scale with respect to $\varepsilon:\left(\boldsymbol{u}_{\perp}, w, p\right) \rightarrow \varepsilon\left(\boldsymbol{u}_{\perp}, w, p\right)$, and so the final form of our governing equations is

$$
\begin{aligned}
& \frac{D}{D t} \boldsymbol{u}_{\perp}=-\nabla_{\perp} p, \\
& \delta^{2} \frac{D w}{D t}=-\frac{\partial p}{\partial z}, \\
& \nabla \cdot \boldsymbol{u}=0,
\end{aligned}
$$

where

$$
\frac{D}{D t} \equiv \frac{\partial}{\partial t}+\varepsilon(\boldsymbol{u} \cdot \nabla)
$$

with

$$
\begin{aligned}
& p=\eta \\
& w=\frac{\partial \eta}{\partial t}+\varepsilon\left(\boldsymbol{u}_{\perp} \cdot \nabla_{\perp}\right) \eta \quad \text { on } \quad z=1+\varepsilon \eta
\end{aligned}
$$

and

$$
w=\left(\boldsymbol{u}_{\perp} \cdot \nabla_{\perp}\right) b \quad \text { on } \quad z=b .
$$

This scaled version of our governing equations immediately permits the identification of the general linear problem; this is simply accomplished by setting $\varepsilon=0$, for arbitrary $\delta$. However, our interests will be mainly focussed on other choices, including the development 
of higher approximations beyond the linear. Indeed, although we will incorporate the parameter $\varepsilon$ in a number of different ways, and $\delta$ may or may not play a critical rôle, we describe only those results which are generated by allowing $\varepsilon \rightarrow 0$. We should note that, at this stage, the equations (2.10)-(2.12) do not obviously allow the accommodation of an arbitrary, ambient 'shear' flow; this will be remedied when we discuss this particular generalisation of the problem.

The technique that we adopt involves the use of asymptotic expansions in one or both parameters. This may require the introduction of suitable scaled variables and then, with or without the need for this further scaling, the resulting equations will be valid only in some region of space and time. Typically, the solution so obtained is uniformly valid in space, at fixed time, particularly since the boundary conditions that we usually impose insist that the wave disturbances decay (often exponentially) at infinity. The validity of the solution for all $t \geq 0$ is, however, more questionable. Most of the analyses that build on, for example, the KdV equation (with suitable initial data which decays sufficiently rapidly at infinity) do appear to produce solutions which are uniformly valid in time, although proofs are not easy to come by. Be this as it may, such issues are rarely of fundamental concern within the context of water waves; the reasons for this are quite clear. Although much of the work on water waves uses an inviscid fluid as the primary model, it is not forgotten that the aim, almost always, is to attempt some understanding of (and, possibly, some predictions for) realisable physical processes. The time scales on which even smallamplitude waves develop and evolve are considerably shorter than those on which the significant effects of viscosity manifest themselves. Thus we can hope to produce useful results for the evolution of the waves, by ignoring viscosity altogether, provided that we do not expect any conclusions to be valid (within the confines of the physical context) for all time. It is therefore the usual practice to require only that the asymptotic solution is valid for times no larger than those for which the appropriate, scaled time is $O(1)$ (as $\varepsilon \rightarrow 0$ and/or $\delta \rightarrow 0$ ); on these grounds we avoid the technically difficult questions of validity as $t \rightarrow \infty$.

Finally, we comment that the governing equations (and the surface boundary conditions, when re-expressed in terms of evaluation on $z=1$ ) possess asymptotic solutions for which all the variables can be represented in the form

$$
q \sim \sum_{n=0}^{\infty} \varepsilon^{n} q_{n} \quad \text { or } \quad q \sim \sum_{n=0}^{\infty} \sum_{m=0}^{\infty} \varepsilon^{n} \delta^{2 m} q_{n m} .
$$

The former choice is to be invoked if the solution is to be constructed for arbitrary, fixed $\delta$ (or, equivalently, $\delta$ has been scaled out of the problem, as is possible in certain circumstances); the latter - a double asymptotic expansion - treats $\varepsilon$ and $\delta$ as independent small parameters. The arguments of the functions $q_{n}$ (and $q_{n m}$ ) are, of course, the appropriate (scaled) variables relevant to the particular problem under consideration.

\section{The Korteweg-de Vries family}

The KdV equation, and all the variants of this equation that we describe, are most conveniently developed in terms of asymptotic expansions in the single parameter $\varepsilon$, having first transformed to remove the parameter $\delta$. This manoeuvre on the governing equations 
is always possible, but not always appropriate: the initial data may depend on $\varepsilon$ and $\delta$ in other ways. The various possible alternatives will be described later. Here, let us scale $\boldsymbol{x}_{\perp}$ and $t$ (as used in equations (2.10)-(2.12)) according to

$$
\boldsymbol{x}_{\perp}=\frac{\delta}{\sqrt{\varepsilon}} \boldsymbol{X}_{\perp}, \quad t=\frac{\delta}{\sqrt{\epsilon}} T
$$

and then, to be consistent with the equation of mass conservation, (2.10), for example, we also require

$$
w=\frac{\sqrt{\epsilon}}{\delta} W
$$

the resulting equations are then $(2.10)-(2.12)$, with $\delta^{2}$ replaced by $\varepsilon$, viz.

$$
\begin{aligned}
& \frac{D}{D T} \boldsymbol{u}_{\perp}=-\widehat{\nabla}_{\perp} p, \\
& \varepsilon \frac{D W}{D T}=-\frac{\partial p}{\partial z}, \\
& \widehat{\nabla} \cdot \boldsymbol{u}=0,
\end{aligned}
$$

where

$$
\frac{D}{D T} \equiv \frac{\partial}{\partial T}+\varepsilon(\boldsymbol{u} \cdot \widehat{\nabla})
$$

with

$$
\begin{aligned}
& p=\eta \\
& W=\frac{\partial \eta}{\partial T}+\varepsilon\left(\boldsymbol{u}_{\perp} \cdot \hat{\nabla}_{\perp}\right) \eta \quad \text { on } \quad z=1+\varepsilon \eta
\end{aligned}
$$

and

$$
W=\left(\boldsymbol{u}_{\perp} \cdot \widehat{\nabla}_{\perp}\right) \widehat{b} \quad \text { on } \quad z=\widehat{b}\left(\boldsymbol{X}_{\perp}\right) ;
$$

the circumflex over the del operators denote that these are now defined in terms of our new variables, (13), and $\widehat{b}\left(\boldsymbol{X}_{\perp}\right)=b\left((\delta / \sqrt{\varepsilon}) \boldsymbol{X}_{\perp}\right)$. We seek a solution of equations (3.3)-(3.5) in the form

$$
q\left(\boldsymbol{X}_{\perp}, z, T ; \varepsilon\right) \sim \sum_{n=0}^{\infty} \varepsilon^{n} q_{n}\left(\boldsymbol{X}_{\perp}, z, T\right), \quad \eta\left(\boldsymbol{X}_{\perp}, T ; \varepsilon\right) \sim \sum_{n=0}^{\infty} \varepsilon^{n} \eta_{n}\left(\boldsymbol{X}_{\perp}, T\right),
$$

where $q$ (and correspondingly $q_{n}$ ) represent the components of $\boldsymbol{u}_{\perp}$ and $W$ and $p$. The two boundary conditions at the surface, (3.4), are then written as conditions on $z=1$, on the assumption that all the relevant functions can be expressed as Taylor series about $z=1$. (This is easily confirmed, at the conclusion of the calculation, to be a valid strategy in this class of problems; the resulting expressions are valid asymptotic expansions as $\varepsilon \rightarrow 0$.)

As our first example, we will obtain the $\mathrm{KdV}$ equation for one-dimensional wave propagation over stationary water of constant depth. Thus we choose to use rectangular Cartesian coordinates, $\boldsymbol{X}_{\perp} \equiv(x, y), \boldsymbol{u}_{\perp} \equiv(u, v)$, and then restrict the wave motion by 
suppressing the appearance of $y$ and $v$. The solution we seek is valid in a suitable region of $(x, t)$-space, defined by (for right-running waves)

$$
\xi=x-T=O(1), \quad \tau=\varepsilon T=O(1)
$$

and it is not necessary that we impose or expect validity outside this region. (Note that we could equally elect to follow left-running waves by introducing $\xi=x+T$.) It is easily confirmed that, to leading order, the initial data required for our equation with $\tau=O(1)$ can be matched back to the corresponding data prescribed at $T=0$ (provided only that it is consistent with the scalings already employed here): the asymptotic representation is uniformly valid for $0 \leq T \leq T_{0}$, with $T_{0}=O\left(\varepsilon^{-1}\right)$. The region of space defined by (3.7) can be interpreted in terms of the original $\boldsymbol{x}_{\perp}$ and $t$, equations (3.1), for any $\delta$, as $\varepsilon \rightarrow 0$. We set $\widehat{b} \equiv 0$, and solve the equations at each order; we find, for example, that

$$
\begin{aligned}
& W \sim-z \eta_{0 \xi}-\varepsilon\left\{\left(\eta_{1 \xi}+\eta_{0 \tau}+\eta_{0} \eta_{0 \xi}+\frac{1}{2} \eta_{0 \xi \xi \xi}\right) z+\frac{1}{6} z^{3} \eta_{0 \xi \xi \xi}\right\}, \quad 0 \leq z \leq 1, \\
& p \sim \eta_{0}+\varepsilon\left\{\eta_{1}+\frac{1}{2}\left(1-z^{2}\right) \eta_{0 \xi \xi}\right\}, \quad 0 \leq z \leq 1,
\end{aligned}
$$

where $\eta_{0}(\xi, \tau)$ satisfies

$$
2 \eta_{0 \tau}+3 \eta_{0} \eta_{0 \xi}+\frac{1}{3} \eta_{0 \xi \xi \xi}=0
$$

(Here, and in most of what follows, we will use subscripts to denote partial derivatives.)

Equation (3.10) is the $\mathrm{KdV}$ equation as it appears in the classical water-wave problem, for the right-running gravity wave. In this far field (defined by $\xi=O(1), \tau=O(1)$ ), the leading term in the representation of the function describing the surface is governed by an equation which allows suitable initial profiles to evolve, essentially by maintaining a balance between nonlinear and dispersive effects. (The derivation that leads to the solution given in (3.8)-(3.10) requires that $u$ (not written down here), $W$ and $p$ exist only by virtue of the passage of the surface wave i.e. if $\eta_{n} \equiv 0, n=0,1, \ldots$, then $u \equiv 0, W \equiv 0$, $p \equiv 0$.) An initial profile, which is prescribed as a function of the original $x$ (i.e. before the use of (3.1)), requires the choice $\delta^{2}=\varepsilon$.

The corresponding problem with cylindrical symmetry can also be formulated; this will describe a surface wave with a circular wave front, which evolves in the presence of nonlinearity and dispersion, on a suitable scale. This requires the choice $\boldsymbol{x}_{\perp} \equiv(r, \theta)$, $\boldsymbol{u}_{\perp} \equiv(u, v)$ so that $\delta^{2}$ is retained, but with $\theta$ and $v$ suppressed; however, there is an additional complication here because a circular wave which moves outwards (or inwards) suffers an amplitude variation by virtue of the changing geometry. The appropriate scaling is therefore more involved than that used for the $\mathrm{KdV}$ equation. Referring to equations (2.10)-(2.12), with $\widehat{b} \equiv 0$ and with the introduction of the polar coordinate $r$, we write (for outward propagating waves)

$$
\xi=\frac{\varepsilon^{2}}{\delta^{2}}(r-t), \quad R=\frac{\varepsilon^{6}}{\delta^{4}} r, \quad(\eta, p, u)=\frac{\varepsilon^{3}}{\delta^{2}}(H, P, U), \quad w=\frac{\varepsilon^{5}}{\delta^{4}} W
$$

and then the equations depend on only the one parameter: $\Delta=\varepsilon^{4} / \delta^{2}$ (i.e. equivalently, $\varepsilon$ and $\delta^{2}$ are each replaced by $\Delta$ ). The structure of the equations, and the asymptotic 
procedure as $\Delta \rightarrow 0$, mirror the pattern already outlined for the KdV equation. The resulting equation, describing the leading term, $H_{0}$, in the representation of the surface wave is the concentric (or cylindrical) $\mathrm{KdV}$ equation (cKdV):

$$
2 H_{0 R}+\frac{1}{R} H_{0}+3 H_{0} H_{0 \xi}+\frac{1}{3} H_{0 \xi \xi \xi}=0
$$

see [35]. The approximation which leads to equation (24) is valid where $\xi=O(1), R=$ $O(1)$, as $\Delta \rightarrow 0$ (but otherwise for any $\varepsilon$ and $\delta$ ); the corresponding inward-moving wave is similarly valid for $R=O(1)$ and so the complications encountered as $R \rightarrow 0$ (where, in general, singularities develop) cannot, strictly, arise here. The initial data for $H_{0}$ is the wave profile at some $R=R_{0}=O(1)$, although it is possible to match as $R \rightarrow 0$, for the outward wave, to the linear solution, since

$$
\eta \sim \frac{1}{\sqrt{r}} f(r-t) \quad \text { as } r \rightarrow \infty
$$

and equation (3.12) has a solution which satisfies

$$
H_{0}(\xi, R) \sim \frac{1}{\sqrt{R}} F(\xi) \quad \text { as } \quad R \rightarrow 0
$$

The inclusion of the second spatial variable, suitably scaled - so, by implication only for special initial profiles - allows the recovery of two-dimensional KdV equations. First, when we choose to use $\boldsymbol{X}_{\perp} \equiv(x, y), \boldsymbol{u}_{\perp} \equiv(u, v)$, and the wave number associated with the profile in the $y$-direction is $O(\sqrt{\varepsilon})$ (for equations (3.3)-(3.5)), we obtain the $2 \mathrm{D} \mathrm{KdV}$, or Kadomtsev-Petviashvili equation; see [29]. The most convenient way to accommodate this small wave number is to scale $y$ itself: we write $Y=\sqrt{\varepsilon} y$ and then, for consistency with the equation of mass conservation, we also require $v=\sqrt{\varepsilon} V$. The equations (3.3)(3.5), with these new variables, together with the variables defined in (3.7) and $\widehat{b} \equiv 0$, produce the equation for the leading term, $\eta_{0}(\xi, Y, \tau)$, as $\varepsilon \rightarrow 0$, in the form

$$
2 \eta_{0 \tau}+3 \eta_{0} \eta_{0 \xi}+\frac{1}{3} \eta_{0 \xi \xi \xi}+V_{0 Y}=0 \quad \text { with } \quad V_{0 \xi}=\eta_{0 Y}
$$

or

$$
\left(2 \eta_{0 \tau}+3 \eta_{0} \eta_{0 \xi}+\frac{1}{3} \eta_{0 \xi \xi \xi}\right)_{\xi}+\eta_{0 Y Y}=0,
$$

the $2 \mathrm{D} \mathrm{KdV}$ equation. The initial data, which may be prescribed at $\tau=0$, is of the form $\eta_{0}(\xi, Y, 0)=\eta_{0}(x, \sqrt{\varepsilon} y, 0)$ which represents a profile, in unscaled variables, which varies slowly in the $y$-direction and therefore the wave front deviates only slowly from the plane wave moving in the $x$-direction. The $2 \mathrm{D} \mathrm{KdV}$ equation has exact solutions which describe obliquely-crossing waves and so, in original $(x, y)$ variables, the solution becomes waves that cross but are almost parallel. (It is illuminating to analyse the problem of waves crossing at any angle, and to use the original $(x, y)$ - unscaled - variables; this solution, for $\varepsilon \rightarrow 0$, is not uniformly valid as the waves become nearly parallel. The appropriate problem for nearly parallel waves recovers precisely our 2D KdV equation; see [36].)

The corresponding extension of the cKdV equation, by allowing a weak ('slow') dependence in the $\theta$-direction, is also possible. We use $\boldsymbol{x}_{\perp} \equiv(r, \theta), \boldsymbol{u}_{\perp} \equiv(u, v)$, and then seek 
a solution which depends on $\Theta$, where $\theta=\sqrt{\Delta} \Theta=\left(\varepsilon^{2} / \delta\right) \Theta$ and $v=\left(\varepsilon^{5} / \delta^{3}\right) V$, and then recalling the definitions (3.11), the surface wave $H \sim H_{0}$ satisfies

$$
\left(2 H_{0 R}+\frac{1}{R} H_{0}+3 H_{0} H_{0 \xi}+\frac{1}{3} H_{0 \xi \xi \xi}\right)_{\xi}+\frac{1}{R^{2}} H_{0 \Theta \Theta}=0
$$

the nearly-concentric KdV (ncKdV) equation.

The four equations obtained so far (KdV, (3.10); cKdV, (3.12); 2D KdV, (3.13); ncKdV, (3.14)) are, through their different representations of the same phenomenon - a surface gravity wave - related. That is, because of the elementary connection between rectangular Cartesian and polar coordinates, and noting that the two equations in $R$ imply a large radius (by virtue of the scalings), we see that two simple transformations exist. Thus the $2 \mathrm{D} \mathrm{KdV}$ equation, written in terms of $\eta_{0}=H(\zeta, \tau), \zeta=\xi+Y^{2} / 2 \tau$, recovers the cKdV equation (after one integration in $\zeta$ and invoking decay conditions at infinity):

$$
2 H_{\tau}+\frac{1}{\tau} H+3 H H_{\zeta}+\frac{1}{3} H_{\zeta \zeta \zeta}=0 .
$$

Similarly, for the ncKdV equation, we write $H=\eta(\zeta, R), \zeta=\xi-(1 / 2) R \Theta^{2}$, then with the same requirement as above on the behaviour at infinity, we find that

$$
2 \eta_{R}+3 \eta \eta_{\zeta}+\frac{1}{3} \eta_{\zeta \zeta \zeta}=0
$$

the $\mathrm{KdV}$ equation. Of course, these special reductions are useful only if the given initial data are consistent with the transformations that we have employed; if not, then we must seek solutions of the original equations. One other useful reduction exists [34], which generalises the one just used above. The choice $H_{0}=\eta(\zeta, R, Y), \zeta=\xi-(1 / 2) R \Theta^{2}$, $Y=R \Theta$, yields the $2 \mathrm{D} \mathrm{KdV}$ equation

$$
\left(2 \eta_{R}+3 \eta \eta_{\zeta}+\frac{1}{3} \eta_{\zeta \zeta \zeta}\right)_{\zeta}+\eta_{Y Y}=0
$$

which immediately extends the class of solutions that are readily available for the ncKdV.

These four examples of familiar KdV-type equations appear as the leading order in the asymptotic representations of the free surface; an important extension - one which plays a significant rôle in our description of the Camassa-Holm equation (Section 8) - is to retain some higher-order terms and truncate. The classic example of this type, in the context of water waves, is the Boussinesq equation, which admits both left- and right-running waves in the one (nonlinear) equation. We return to equations $(3.3)-(3.5)$, set $\widehat{b} \equiv 0$ and introduce $\boldsymbol{X}_{\perp} \equiv(x, y), \boldsymbol{u}_{\perp} \equiv(u, v)$, but allow only one-dimensional propagation. However, we do not scale the variables further: the approximation that we develop is valid for $x=O(1), T=O(1)$, as $\varepsilon \rightarrow 0$. Seeking asymptotic expansions as before, we find that $\eta_{0}(x, T)$ and $\eta_{1}(x, T)$ satisfy, respectively, the equations

$$
\eta_{0 T T}-\eta_{0 x x}=0, \quad \eta_{1 T T}-\eta_{1 x x}-\left(\frac{1}{2} \eta_{0}^{2}+u_{0}^{2}\right)_{x x}-\frac{1}{3} \eta_{0 x x x x}=0,
$$

where

$$
u_{0}(x, T)=-\int_{-\infty}^{x} \eta_{0 T} d x
$$


(on the assumption that $u_{0} \rightarrow 0$ as $x \rightarrow-\infty$ ). These two equations, (3.15) and (3.16), enable us to form a single equation for $\eta=\eta_{0}+\varepsilon \eta_{1}+O\left(\varepsilon^{2}\right)$ :

$$
\eta_{T T}-\eta_{x x}-\varepsilon\left\{\frac{1}{2} \eta^{2}+\left(\int_{-\infty}^{x} \eta_{T} d x\right)^{2}\right\}_{x x}-\frac{1}{3} \varepsilon \eta_{x x x x}=O\left(\varepsilon^{2}\right) .
$$

This equation is more conveniently written in a Lagrangian rather than an Eulerian frame, by setting

$$
H=\eta-\varepsilon \eta^{2}, \quad X=x+\varepsilon \int_{-\infty}^{x} \eta(x, T ; \varepsilon) d x
$$

and then the equation for $H(X, T ; \varepsilon)$ becomes

$$
H_{T T}-H_{X X}-\frac{3}{2} \varepsilon\left(H^{2}\right)_{X X}-\frac{1}{3} \varepsilon H_{X X X X}=O\left(\varepsilon^{2}\right) .
$$

This equation is the Boussinesq equation and, with the omission of the error term on the right, it is completely integrable for $\forall \varepsilon>0$. (It is usual, therefore, to set the righthand side to zero and then to scale out $\varepsilon$ altogether.) The equation possesses solutions, most particularly solitons, which may propagate either all in the same direction (as for the $\mathrm{KdV}$ equation) or in opposite directions, producing head-on soliton collisions.

Finally, we observe that the inclusion of a weak dependence on the $y$-coordinate, exactly as required for the $2 \mathrm{D} \mathrm{KdV}$ equation, (3.13), in a Boussinesq derivation, produces the 2D Boussinesq equation:

$$
H_{T T}-H_{X X}-\frac{3}{2} \varepsilon\left(H^{2}\right)_{X X}-\frac{1}{3} \varepsilon H_{X X X X}-\varepsilon H_{Y Y}=0 .
$$

This equation, however, is not completely integrable; although it does possess a general 2 -soliton solution, it does not have a 3-soliton counterpart - only special 3-soliton solutions exist $[18,24]$.

\section{Extending the $\mathrm{KdV}$ model}

Here our primary interest is in how the KdV equation, and related equations, manifest themselves in water-wave problems that attempt a more accurate and comprehensive description of real phenomena. Thus we could examine the effects of including, for example, an underlying shear flow, or variable depth (or both). Further, these descriptions would provide only the first approximations for the surface wave; higher-order terms (which might represent various reflected and re-reflected waves) could then be investigated. Here, we will simply outline what happens when either a shear flow, or variable depth, is included in the model.

In the case of an underlying, ambient shear flow, we first require an adjustment to our governing equations, (3.3)-(3.5). We will consider only one-dimensional propagation (in the $x$-direction) and then we replace

$$
\varepsilon \boldsymbol{u}_{\perp} \equiv \varepsilon(u, v) \rightarrow\{U(z)+\varepsilon u, 0\},
$$


where $U(z)$ is the prescribed velocity profile in the absence of any waves. The solution that we seek will be expressed in terms of the variables

$$
\xi=x-c T=O(1), \quad \tau=\varepsilon T=O(1),
$$

where $c$ is a constant to be determined; cf. (3.7). The asymptotic solution, (3.6), then yields, to leading order,

$$
p_{0}=\eta_{0}, \quad W_{0}=[U(z)-c] I_{2}(z) \eta_{0 \xi}, \quad u_{0}=-\eta_{0} \frac{d}{d z}\left[\{U(z)-c\} I_{2}(z)\right],
$$

all for $0 \leq z \leq 1$, where

$$
I_{2}(z)=\int_{0}^{z} \frac{d z}{[U(z)-c]^{2}}
$$

The speed of the waves, $c$, is given by the solution(s) of

$$
I_{2}(1)=1 \quad \text { i.e. } \quad \int_{0}^{1} \frac{d z}{[U(z)-c]^{2}}=1
$$

this is the Burns condition [5]. For monotonic profiles, $U(0) \leq U(z) \leq U(1)$, two solutions always exist: $c>U(1)$ and $c<U(0)$. There may be, in addition, critical-layer solutions: $c=U\left(z_{c}\right), 0<z_{c}<1$; these are solutions of the integral, in (4.3), interpreted as the Cauchy Principal Value. (The Burns condition appears in this form for problems in which critical layers are present, when solutions are constructed, separately, in $0 \leq z<z_{c}$ and in $z_{c}<z \leq 1$; see $[3,33,21,22]$.) We will assume, in this discussion, that the solution for $c$ does not correspond to a critical layer (and we note in passing that the case $U \equiv 0$ recovers $c= \pm 1$, the familiar speeds for right- and left-running waves).

At the next order, we obtain the $\mathrm{KdV}$ equation for $\eta_{0}(\xi, \tau)$ :

$$
-2 I_{31} \eta_{0 \tau}+3 I_{41} \eta_{0} \eta_{0 \xi}+J_{1} \eta_{0 \xi \xi \xi}=0
$$

where

$$
\begin{aligned}
& I_{n 1}=I_{n}(1)=\int_{0}^{1} \frac{d z}{[U(z)-c]^{n}} \text { and } \\
& J_{1}=\int_{0}^{1} \int_{z}^{1} \int_{0}^{\zeta} \frac{[U(\zeta)-c]^{2}}{[U(z)-c]^{2}[U(Z)-c]^{2}} d Z d \zeta d z .
\end{aligned}
$$

This is an encouraging result: no matter the form of the flow below the surface, there is a region of $(x, T)$-space where the $\mathrm{KdV}$ equation exists. Thus, in nature, we might reasonably expect to see, on suitable scales, solitary waves and solitons on the surface of moving water.

We now turn to the problem of allowing the depth of the water to vary; this situation gives rise to a less satisfactory outcome in terms of integrable equations, but the conclusion is not that surprising. We revert to equations (3.3)-(3.5) (so the water is stationary in the absence of waves), consider propagation only in the positive $x$-direction $\left(\boldsymbol{X}_{\perp} \equiv(x, y)\right)$, and introduce $\widehat{b}=\widehat{b}(x)$. An issue in problems of this type is: on what scale should we allow the depth to vary? Let us write $\widehat{b}=B(\alpha x)$, then there are three distinctly different cases: 
$\alpha=o(\varepsilon) ; \alpha=O(\varepsilon) ; \varepsilon=o(\alpha)$. The mathematically most interesting situation occurs when the depth changes on the same scale as the wave evolves i.e. $\alpha=O(\varepsilon)$. Thus we write $\widehat{b}=B(\varepsilon x)$ (and then the bottom boundary condition, (3.5), becomes $W=\varepsilon u B^{\prime}(\varepsilon x)$ on $z=B(\varepsilon x))$ and introduce the variables

$$
\xi=\varepsilon^{-1} f(X)-T, \quad X=\varepsilon x
$$

where it is more convenient to work with large distance rather than large time. The function $f(X)$ accommodates the expected variable speed of the waves as $B$ changes (and $\partial \xi / \partial x=O(1)$, as required by the governing equations, so that the constant-depth case is recovered by the choice $f(X)=X$ i.e. $\xi=X / \varepsilon-T=x-T)$. The leading order now produces

$$
p_{0}=\eta_{0}, \quad u_{0}=\eta_{0} f^{\prime}(X), \quad W_{0}=\eta_{0 \xi}(B-z)\left(f^{\prime}(X)\right)^{2}, \quad 0 \leq z \leq 1,
$$

with

$$
f(X)=\int_{0}^{X} \frac{d X}{\sqrt{D(X)}},
$$

where $D(X)=1-B(X)(>0)$ is the local depth. (We have selected the positive square root, for rightward propagation.) At the next order we generate a KdV-type equation for $\eta_{0}(\xi, X)$ :

$$
2 \sqrt{D} \eta_{0 X}+\frac{D^{\prime}(X)}{2 \sqrt{D}} \eta_{0}+\frac{3}{D} \eta_{0} \eta_{0 \xi}+\frac{D}{3} \eta_{0 \xi \xi \xi}=0
$$

or

$$
2 H_{0 X}+\frac{3}{D^{7 / 4}} H_{0} H_{0 \xi}+\frac{D^{1 / 2}}{3} H_{0 \xi \xi \xi}=0, \quad H_{0}(\xi, X)=(D(X))^{1 / 4} \eta_{0}(\xi, X) .
$$

This variable-depth KdV equation is, in general, not completely integrable; but it clearly is with $D \equiv 1$ - and in this sense our new equation is nearly integrable. (Another special case occurs with $\eta_{0}=D^{2} H\left(\xi, \int \sqrt{D} d X\right)$, with $D=(a X+b)^{9 / 4}, a$ and $b$ arbitrary constants; this recovers the $\mathrm{cKdV}$ equation.)

The majority of the investigations, for variable depth, have been for the case $\alpha=o(\varepsilon)$, but with $\alpha$ otherwise treated as another parameter in the problem. Then equation (4.7) becomes the KdV equation, with $D^{\prime}$ absent - this term appears now at higher order and $D$ dependent on a third variable within a multiple-scale formulation of the equations. The efforts have then been directed at discovering all the wave components (reflected waves, etc.) which contribute, for example, to the overall mass balance; see [36, 30, 31, 23]. It is a fairly straightforward exercise to analyse the even more general problem of an ambient shear flow which varies as the water flows in a variable-depth environment; the modifications of the classical $\mathrm{KdV}$ equation that are evident in equations (4.4) and (4.7) are combined in this new problem; see [25]. 


\section{The nonlinear Schrödinger family}

Initial data, for the surface wave, which takes the form

$$
A(\varepsilon x) \exp (i k x)+c . c .
$$

where c.c. denotes the complex conjugate, and is associated with our equations which contain $\delta$ explicitly, (2.10)-(2.12), for arbitrary (fixed) $\delta$, generate the NLS equation. We seek a solution which represents an amplitude modulation of a harmonic wave, the modulation being described by the variables

$$
\zeta=\varepsilon\left(x-c_{g} t\right), \quad \tau=\varepsilon^{2} t,
$$

where $c_{g}(k)$ is the group speed, with wave number $k$. The carrier wave is described by $\xi=x-c_{p} t$, where $c_{p}(k)$ is the phase speed. We seek an asymptotic solution, in the usual format, as $\varepsilon \rightarrow 0$ (at fixed $\delta$ ), with

$$
\eta_{0}(\xi, \zeta, \tau)=A_{0}(\zeta, \tau) \exp (i k \xi)+c . c . .
$$

The higher-order terms, $\eta_{n}$, require the inclusion of higher harmonics (for the existence of a periodic solution); indeed, we find that

$$
\eta_{n}=\sum_{m=0}^{n+1} A_{n m}(\zeta, \tau) E^{m}+c . c ., \quad E=\exp (i k \xi), \quad n=1,2, \ldots
$$

Working as far as terms of $O\left(\varepsilon^{2}\right)$, and collecting the various terms $E^{m}$, we find that

$$
c_{p}^{2}=\frac{\tanh (\delta k)}{\delta k}, \quad c_{g}=\frac{1}{2} c_{p}(1+2 \delta k \operatorname{cosech}(2 \delta k)),
$$

which confirms the familiar identity $c_{g}=d \omega / d k$, where $\omega=k c_{p}$. The calculation is lengthy, and rather tedious, but eventually produces the equation for $A_{0}$ :

$$
-2 i k c_{p} A_{0 \tau}-k c_{p} \frac{d^{2} \omega}{d k^{2}} A_{0 \zeta \zeta}+\beta A_{0}\left|A_{0}\right|^{2}=0,
$$

where

$$
\begin{aligned}
\beta= & \frac{k^{2}}{c_{p}^{2}}\left\{\frac{1}{2}\left(1+9 \operatorname{coth}^{2} \delta k-13 \operatorname{sech}^{2} \delta k-2 \tanh ^{4} \delta k\right)\right. \\
& \left.-\left(2 c_{p}+c_{g} \operatorname{sech}^{2} \delta k\right)^{2}\left(1-c_{g}^{2}\right)^{-1}\right\} ;
\end{aligned}
$$

this equation, (5.4), is the NLS equation. Of some interest, and relevance, is the observation in this equation that the coefficient of the term $A_{0 \zeta \zeta}$ is always positive, but that $\beta$ changes sign across $\delta k=\delta k_{0} \approx 1.363$; for $k>k_{0}$ we have $\beta>0$ and this corresponds to the classical wave packet associated with observed wave motion. If $\beta<0$ then the solution becomes the so-called dark soliton, and this is not applicable to water waves if there are undisturbed conditions at infinity. 
The extension to two dimensions is easily accomplished, simply by allowing the initial profile to take the form

$$
A(\varepsilon x, \varepsilon y) \exp (i k x)+c . c .,
$$

and we note that, here, the slow (weak) variation in the amplitude function is on the same scale in both $x$ and $y$. We introduce $Y=\varepsilon y$ and follow the procedure outlined for the NLS equation; this time we obtain

$$
\begin{aligned}
& -2 i k c_{p} A_{0 \tau}-k c_{p} \frac{d^{2} \omega}{d k^{2}} A_{0 \zeta \zeta}-c_{p} c_{g} A_{0 Y Y} \\
& +\left\{\beta+\frac{\gamma^{2} k^{2}}{c_{p}^{2}\left(1-c_{g}^{2}\right)}\right\} A_{0}\left|A_{0}\right|^{2}+\gamma k^{2} A_{0} f_{0 \zeta}=0
\end{aligned}
$$

with

$$
\left(1-c_{g}^{2}\right) f_{0 \zeta \zeta}+f_{0 Y Y}=-\frac{\gamma}{c_{p}^{2}}\left(\left|A_{0}\right|^{2}\right)_{\zeta},
$$

where

$$
\gamma=2 c_{p}+c_{g} \operatorname{sech}^{2} \delta k
$$

Equations (5.5) and (5.6) are the Davey-Stewartson (DS) equations [9]; these equations form an integrable pair in the case of long waves $(\delta \rightarrow 0)$ (see [1]).

\section{Matching KdV and NLS families}

The NLS and DS equations are valid for arbitrary $\delta$, as $\varepsilon \rightarrow 0$; the KdV equations are valid for arbitrary $\delta$, again as $\varepsilon \rightarrow 0$, but the variables (and hence region of validity) are different, because the variables are not scaled in the same way. To compare the two, we must first remove the scaling, (3.1), and revert to the original $\left(\boldsymbol{x}_{\perp}, t\right)$ variables; our various $\mathrm{KdV}$ equations now contain the parameter $\delta^{2} / \varepsilon$ and we require $\delta^{2} / \varepsilon=O(1)$ for the consistency of the equations in this approximation. Thus, for example, the KdV equation (3.10) becomes

$$
2 \eta_{0 \widehat{\tau}}+3 \eta_{0} \eta_{0 \widehat{\xi}}+(\lambda / 3) \eta_{0 \widehat{\xi} \widetilde{\xi} \xi}=0
$$

where $\widehat{\xi}=(\delta / \sqrt{\varepsilon}) \xi, \widehat{\tau}=(\delta / \sqrt{\varepsilon}) \tau$ and $\lambda=\delta^{2} / \varepsilon$. Because we have taken $\varepsilon \rightarrow 0$, we therefore require $\delta^{2}=O(\varepsilon)$; but the NLS equation is valid for $\delta=O(1)$, when written in essentially the same (unscaled) variables for the carrier wave. Thus we might expect that the NLS equation with $\delta \rightarrow 0$ would match to an appropriate solution of the KdV equation with $\delta \rightarrow \infty$ (i.e. $\lambda \rightarrow \infty$ ).

First, the NLS equation, (5.4), is approximated as $\delta \rightarrow 0$, to give

$$
-2 i k \delta^{2} A_{0 \tau}+\delta^{4} k^{2} A_{0 \zeta \zeta}-\frac{9}{2} A_{0}\left|A_{0}\right|^{2}=0,
$$

where we have used

$$
c_{p} \sim 1-\frac{1}{6} \delta^{2} k^{2} \quad \text { and } \quad c_{g} \sim 1-\frac{1}{2} \delta^{2} k^{2} .
$$


Now we seek a solution of equation (6.1) in the form

$$
\eta_{0} \sim \sum_{n=0}^{\infty} \lambda^{-n}\left\{\sum_{m=0}^{n+1} A_{n m}(Z, T) \exp (i k m \Xi)+c . c\right\}
$$

where

$$
\Xi=\widehat{\xi}+C_{p} \lambda \widehat{\tau}, \quad Z=\lambda^{-1}\left(\widehat{\xi}+C_{g} \lambda \widehat{\tau}\right), \quad T=\lambda^{-1} \widehat{\tau}
$$

we find that $C_{p}=k^{2} / 6, C_{g}=k^{2} / 2$, and $A_{01}$ satisfies

$$
-2 i k A_{01 T}+k^{2} A_{01 Z Z}-\frac{9}{2} A_{01}\left|A_{01}\right|^{2}=0 .
$$

The definitions that we have introduced here imply that $\tau=\delta^{2} T$ and $\zeta=\delta^{2} Z$ and so (6.2) becomes, written in these variables

$$
-2 i k A_{0 T}+k^{2} A_{0 Z Z}-\frac{9}{2} A_{0}\left|A_{0}\right|^{2}=0
$$

which is identical to equation (6.4), when $A_{0}$ is interpreted as $A_{01}$. Thus the short-wave limit of the KdV equation, for modulated harmonic waves, matches to the long-wave limit of the NLS equation. This same matching property also exists between the DS and 2D KdV equations; see [15].

\section{Extending the NLS model}

There are three extensions to the basic NLS equation, within the water-wave context, that we briefly mention; these involve the introduction of an underlying shear flow, the presence of variable depth and - a novel exercise - the character of the problem near the zero of $\beta$ (see (5.4)). First, let the shear flow be (as before) given as $u=U(z)=O(1)$, then the development which culminates in the appearance of the relevant NLS equation involves various integrals expressed in terms of the solution, $F(z)$, of the problem

$$
\frac{d}{d z}\left\{\frac{1}{\left[U(z)-c_{p}\right]^{2}} \frac{d F}{d z}\right\}-\left(\frac{\delta k}{U(z)-c_{p}}\right)^{2} F=0
$$

with $F(1)=1, F^{\prime}(0)=0$; a third condition, $F^{\prime}(1)=(\delta k)^{2}\left[U(1)-c_{p}\right]$, gives $c_{p}$ as the solution of

$$
\int_{0}^{1} \frac{F(z)}{\left[U(z)-c_{p}\right]^{2}} d z=1
$$

a generalisation of the Burns condition, (4.3). The method of solution follows that for the classical NLS equation, with the additional complication of the dependence on $U(z)$; the details are given in [19]. The NLS equation for $A_{0}$, now with shear, takes precisely the same form as before (see (5.4)), namely

$$
-2 i k \widehat{\alpha} A_{0 \tau}+\widehat{\gamma} A_{0 \zeta \zeta}+\widehat{\beta} A_{0}\left|A_{0}\right|^{2}=0,
$$


where $\widehat{\alpha}, \widehat{\beta}$ and $\widehat{\gamma}$ are involved functions of $k$, containing numerous integrals in $U(z)$. The important observation is that, as for the corresponding $\mathrm{KdV}$ problem, the existence of a modulated harmonic wave, governed by the NLS equation, occurs even in the presence of arbitrary shear flows.

The inclusion of variable depth, but in the absence of any shear flow, follows the same philosophy as we adopted for the KdV equation [11]. Thus we suppose that the depth varies on the same scale as that for time (i.e. $\varepsilon^{-2}$ ), but we elect to use the spatial rather than the temporal variable. To accommodate the variable depth, we introduce

$$
X=\varepsilon^{2} x, \quad \zeta=\varepsilon\left(\varepsilon^{-2} \int_{0}^{X} c_{g}^{-1}(X, k) d X-t\right)
$$

and describe the carrier wave (for fixed $k$ ) and

$$
E=\exp (i \phi), \quad \frac{\partial \phi}{\partial x}=k, \quad \frac{\partial \phi}{\partial t}=-k c_{p}(X, k) .
$$

The amplitude of the first harmonic (the leading term in the asymptotic expansion) is then a solution of the NLS-type equation:

$$
-2 i k c_{p} c_{g} A_{X}-i k^{2} c_{p}^{2} A \frac{\partial\left(c_{g} / \omega\right)}{\partial X}-\frac{k c_{p}}{c_{g}^{2}} \frac{d^{2} \omega}{d k^{2}} A_{\zeta \zeta}+\widetilde{\beta} A|A|^{2}=0 .
$$

The coefficients depend on $X$, through the local depth $D(X)=1-B(X)$, with

$$
c_{p}^{2}=\frac{\tanh \delta k D}{\delta k}, \quad c_{g}=\frac{1}{2} c_{p}(1+2 \delta k D \operatorname{cosech} 2 \delta k D), \quad \omega=k c_{p},
$$

and $\widetilde{\beta}$ is $\beta$ (see (5.4)) with $\delta$ replaced by $\delta D(X)$. This equation is more compactly written in terms of $\widetilde{A}=A \sqrt{c_{g} / \omega}$ :

$$
-2 i k c_{p} c_{g} \widetilde{A}_{X}-\frac{k c_{p}}{c_{g}^{2}} \frac{d^{2} \omega}{d k^{2}} \widetilde{A}_{\zeta \zeta}+\frac{\omega \widetilde{\beta}}{c_{g}} \widetilde{A}|\widetilde{A}|^{2}=0,
$$

which becomes the conventional NLS equation, in the context of water waves, when $D=1$ : another 'nearly-integrable' equation.

Finally, we mention what happens in the situation where $k$ is close to $k_{0}$ - the value at which $\beta=0$ - in equation (5.4). In order to balance the time evolution and (quintic) nonlinearity, we require (cf. (5.2)) the scales $T=\varepsilon^{4} t, Z=\varepsilon^{2}\left(x-c_{g} t\right)$, and then, with $\beta=0$, we obtain the equation

$$
\begin{aligned}
& 2 i A_{0 T}-\delta a_{1} A_{0 Z Z}+k a_{2} A_{0}\left|A_{0}\right|^{4} \\
& \quad+i\left(a_{3}\left|A_{0}\right|^{2} A_{0 Z}-a_{4} A_{0}\left(\left|A_{0}\right|^{2}\right)_{z}\right)-k a_{5} A_{0} \psi_{0 T}=0,
\end{aligned}
$$

where the $a_{i}>0$ are constants (corresponding to evaluation on $\delta k=\delta k_{0} \approx 1.363$ ) and $\psi_{0 Z}=\left|A_{0}\right|^{2}$. A uniformly valid equation, valid for $\beta=O(1)$ and smaller, can be obtained by combining the classical NLS equation, (5.4), with the above; see [20]. The resulting equation can be used to examine, for example, the stability of the Stokes wave, an important aspect of classical water-wave theory; see [2]. 


\section{The Camassa-Holm equation}

We complete this overview of the various completely integrable (and closely related) equations that appear in water-wave theory by describing the more recent investigations surrounding the $\mathrm{CH}$ equation. After its introduction (implied in [14]), and its further properties and importance explored [6,7], the rôle of the $\mathrm{CH}$ equation in water waves became an issue. (The method for integrating the $\mathrm{CH}$ equation can be found in [8], and explicit soliton solutions are given in [27].) We outline how this equation can be obtained, following a systematic procedure, from our governing equations; the details can be found in [26] (and a similar idea is included in [13]). We start with equations $(2.10)-(2.12)$ (with $b \equiv 0)$, introduce $\boldsymbol{x}_{\perp} \equiv(x, y), \boldsymbol{u}_{\perp}=(u, v)$, but consider propagation in only the $x$-direction. In this approach, we expand in a double asymptotic expansion, valid for $\varepsilon \rightarrow 0, \delta \rightarrow 0$, after scaling according to

$$
\zeta=\sqrt{\varepsilon}(x-t), \quad \tau=\varepsilon \sqrt{\varepsilon} t, \quad w=\sqrt{\varepsilon} W
$$

(for right-running waves). We find that $\eta \sim \eta_{00}+\varepsilon \eta_{10}+\delta^{2} \eta_{01}+\varepsilon \delta^{2} \eta_{11}$ satisfies a higher order $\mathrm{KdV}$ equation

$$
2 \eta_{\tau}+3 \eta \eta_{\zeta}+\frac{1}{3} \delta^{2} \eta_{\zeta \zeta \zeta}-\frac{3}{4} \varepsilon \eta^{2} \eta_{\zeta}=-\frac{1}{12} \varepsilon \delta^{2}\left(23 \eta_{\zeta} \eta_{\zeta \zeta}+10 \eta \eta_{\zeta \zeta \zeta}\right)+O\left(\varepsilon^{2}, \delta^{4}\right)
$$

and that, to the same order,

$$
u \sim \eta-\frac{1}{4} \varepsilon \eta^{2}+\varepsilon \delta^{2}\left(\frac{1}{3}-\frac{1}{2} z^{2}\right) \eta_{\zeta \zeta}
$$

The $\mathrm{CH}$ equation, it transpires, describes the $x$-component, $u$, of the velocity in the flow at a certain depth below the surface. To see this, let us first write the evaluation at depth $z=z_{0}\left(0 \leq z_{0} \leq 1\right)$ as $\widehat{u}$, and then we invert equation (8.3) to give

$$
\eta \sim \widehat{u}+\frac{1}{4} \varepsilon \widehat{u}^{2}-\varepsilon \delta^{2} \lambda \widehat{u}_{\zeta \zeta}
$$

where $\lambda=\frac{1}{3}-\frac{1}{2} z_{0}^{2}$. This is used in equation (8.2) to give

$$
2 \widehat{u}_{\tau}+3 \widehat{u}_{\zeta}+\frac{1}{3} \delta^{2} \widehat{u}_{\zeta \zeta \zeta}=-\varepsilon \delta^{2}\left\{\left(6 \lambda+\frac{29}{12}\right) \widehat{u}_{\zeta} \widehat{u}_{\zeta \zeta}+\frac{5}{6} \widehat{u}_{\zeta \zeta \zeta}\right\}+O\left(\varepsilon^{2}, \delta^{4}\right)
$$

but, in order to recover a $\mathrm{CH}$ equation, we must work with the original $(x, t)$-variables, scaled appropriately. Thus we write $Z=\sqrt{\varepsilon} x, T=\sqrt{\varepsilon} t$, use these in (8.4) and add the term $\varepsilon \delta^{2} \mu\left(\widehat{u}_{Z Z T}-\widehat{u}_{Z Z T}\right)$ (for any arbitrary, real constant $\mu$ ); when we incorporate the approximation $\widehat{u}_{T} \sim-\left(\widehat{u}_{Z}+\frac{3}{2} \varepsilon \widehat{u}_{Z}\right)$ into one of these terms, we obtain

$$
\begin{aligned}
2\left(\widehat{u}_{T}\right. & \left.+\widehat{u}_{Z}\right)+3 \varepsilon \widehat{u}_{Z}+\varepsilon \delta^{2}\left(\frac{1}{3}-\mu\right) \widehat{u}_{Z Z Z}-\varepsilon \delta^{2} \mu \widehat{u}_{Z Z T} \\
& =-\varepsilon^{2} \delta^{2}\left\{\left(6 \lambda-\frac{9}{2} \mu+\frac{29}{12}\right) \widehat{u}_{Z} \widehat{u}_{Z Z}+\left(\frac{5}{6}-\frac{3}{2} \mu\right) \widehat{u}_{Z Z Z}\right\}+O\left(\varepsilon^{3}, \varepsilon \delta^{4}\right) .
\end{aligned}
$$


This equation becomes, in all its essentials, a CH equation if we choose $\mu=\frac{1}{2}+4 \lambda$ and $\mu=5 / 6$; indeed, it takes the standard form when we write it in terms of

$$
\begin{aligned}
& X=2 \sqrt{\frac{5}{3}}\left(Z-\frac{3}{5} T\right), \quad \widehat{u}=\sqrt{\frac{3}{5}} U(X, T ; \varepsilon, \delta): \\
& U_{T}+2 \kappa U_{X}+3 \varepsilon U U_{X}-\varepsilon \delta^{2} U_{X X T}=\varepsilon^{2} \delta^{2}\left(2 U_{X} U_{X X}+U U_{X X X}\right),
\end{aligned}
$$

where $2 \kappa=(4 / 5) \sqrt{3 / 5}$. This is a CH equation, with error $O\left(\varepsilon^{3}, \varepsilon \delta^{4}\right)$; it is valid at the depth $z_{0}=1 / \sqrt{2}$. The solution of this equation then gives, to this order of approximation, the surface wave as

$$
\eta \sim \sqrt{\frac{5}{3}}\left(U+\frac{1}{4} \sqrt{\frac{5}{3}} \varepsilon U^{2}-\frac{1}{5} \varepsilon \delta^{2} U_{X X}\right),
$$

which is (but only to this order of approximation) a solution of equation (8.2). Now we are in a position to explore the ways in which the $\mathrm{CH}$ equation is relevant to other, more general problems in water waves.

For example, we may allow a (suitably weak) dependence on the $y$-coordinate; if we introduce $Y=\varepsilon y, v=\varepsilon V$, (but other choices are possible) then we obtain the simplest 2D $\mathrm{CH}$ equation:

$$
\left(U_{T}+2 \kappa U_{X}+3 \varepsilon U U_{X}-\varepsilon \delta^{2} U_{X X T}\right)_{X}+\varepsilon^{2} U_{Y Y}=\varepsilon^{2} \delta^{2}\left(2 U_{X} U_{X X}+U U_{X X X}\right)_{X}
$$

with the same error terms as before (see (8.5)).

However, the problem of incorporating an underlying shear is a much more demanding exercise. Indeed, the choice of a general $U(z)$ has yet to be completed - and its complexity is such that it is far from clear what would be learnt. Fortunately, the nature of the problem is, we believe, fairly readily gauged by examining the case of a linear shear: $U(z)=\alpha z$ $(\alpha \geq 0)$ [28]. The procedure is that outlined above, except that we must use $\zeta=\sqrt{\varepsilon}(x-c t)$, where $c$ is a solution of the Burns condition

$$
\int_{0}^{1} \frac{d z}{[\alpha z-c]^{2}}=1 \quad \text { i.e. } \quad c=\frac{1}{2}\left(\alpha \pm \sqrt{\alpha^{2}+4}\right) .
$$

(We note that $c= \pm 1$ when $\alpha=0$ : the familiar right- and left-going waves in the elementary theories.) The equation for $U$ (i.e. $u$ evaluated at $z=z_{0}$; see (8.5)) cannot be recast as a $\mathrm{CH}$ equation if $c \neq \pm 1$; here we require the equation for

$$
v=U+\varepsilon \beta U^{2}+O\left(\varepsilon^{2}, \delta^{4}\right)
$$

where $\beta(c)$ is to be determined. (It is more convenient, and neater, to use $c$ rather than $\alpha$ as the parameter which describes the linear shear; note that $\beta( \pm 1)=0$.) The equation for $v$ (after suitable scalings that mirror what we did above) is the standard $\mathrm{CH}$ equation, (8.5), with

$$
\beta=\frac{c^{5}\left(c^{4}+c^{2}-2\right)}{2\left(c^{4}+c^{2}+1\right)\left(1+c^{2}\right)^{2}}, \quad 2 \kappa=\frac{2 \sqrt{2}}{3 c}\left(1+c^{2}\right)^{2}\left(\frac{c^{4}+c^{2}+1}{c^{4}+6 c^{2}+3}\right)^{3 / 2}
$$


and $U$ is defined at the depth

$$
z_{0}=\frac{\sqrt{2 c^{12}+16 c^{10}+33 c^{8}+31 c^{6}+32 c^{4}-3 c^{2}-3}}{\sqrt{6}\left(1+c^{2}\right)\left(c^{4}+c^{2}+1\right)} .
$$

This depth recovers the previous result for $c= \pm 1\left(z_{0}=1 / \sqrt{2}\right)$ - we expect upstream and downstream propagation to be the same for no shear - but it is not defined for $\forall \alpha \geq 0$. Although a depth, $0<z_{0}<1$, always exists for downstream propagation $(c \geq 1)$, this is not the situation for the upstream case. For $0>c>c_{0}, c_{0} \approx-0.544(\alpha \approx 1.296), z_{0}$ is not real: the depth at which the $\mathrm{CH}$ equation is valid does not exist within the flow field. The consequences of this, both for the flow as a whole and for the surface wave in particular, are currently being investigated.

Finally, we mention one very new observation about $\mathrm{CH}$ equations in water waves. Recently, Degasperis, Holm \& Hone [10] have found a second completely integrable CHtype equation; it takes the form

$$
u_{t}+2 \kappa u_{x}+4 u u_{x}-u_{x x t}=3 u_{x} u_{x x}+u u_{x x x} .
$$

This equation can also be generated within the water-wave problem (with no shear and $b \equiv 0)$. In this case, we find that $2 \kappa=\frac{21}{40} \sqrt{\frac{3}{10}}$ and the depth at which $U$ is defined (see $(8.5))$ is now $\frac{1}{3} \sqrt{\frac{11}{2}}$.

\section{Summary}

This presentation has attempted to give an overview of the techniques employed, and the results obtained, when the aim is to seek completely integrable equations within the classical water-wave problem. Many variants of these equations have been derived, including some which are, at best, 'nearly' integrable. Some relations between these equations, both in the sense of exact transformations and via matching, have been demonstrated. An indication has been given of the rôle of these equations in the wider context of water waves, and in nonlinear wave propagation more generally. The recent work on the Camassa-Holm equation has suggested some intriguing lines of investigation, and these are currently being pursued.

\section{References}

[1] Anker D and Freeman N C, On the Soliton Solutions of the Davey-Stewartson Equations for Long Waves, Proc. Roy. Soc. A360 (1978), 529-540.

[2] Benjamin T B and Feir J E, The Disintegration of Wave Trains on Deep Water, I: Theory, J. Fluid Mech. 27 (1967), 417-430.

[3] Benney D J and Bergeron R F, A New Class of Nonlinear Waves in Parallel Flows, Stud. Appl. Math. 48 (1969), 181-204.

[4] Boussinesq J, Théorie de l'intumescence liquid appelée onde solitaire ou de translation, se propageant dans un canal rectanulaire, Comptes Rendus Acad. Sci. (Paris) 72 (1871), $755-759$. 
[5] Burns J C, Long Waves in Running Water, Proc. Camb. Phil. Soc. 49 (1953), 695-706.

[6] Camassa R and Holm D D, An Integrable Shallow Water Equation with Peaked Solitons, Phys. Rev. Lett. 71 (1993), 1661-1664.

[7] Camassa R, Holm D D and Hyman J M, A New Integrable Shallow Water Equation, Adv. Appl. Mech. 31 (1994), 1-33.

[8] Constantin A, On the Scattering Problem for the Camassa-Holm Equation, Proc. Roy. Soc. A457 (2001), 953-970.

[9] Davey A and Stewartson K, On Three-Dimensional Packets of Surface Waves, Proc. Roy. Soc. A338 (1974), 101-110.

[10] Degasperis A, Holm D D and Hone A N W, A New Integrable Equation with Peakon Solutions, Theoret. Math. Phys. (2002), to appear.

[11] Djordjevic V D and Redekopp L G, On the Development of Packets of Surface Gravity Waves Moving over an Uneven Bottom, J. Appl. Math. Phys. 29 (1978), 950-962.

[12] Drazin P G and Johnson R S, Solitons: An Introduction, Cambridge University Press, 1992.

[13] Dullin H R, Gottwald G and Holm D D, An Integrable Shallow Water Equation with Linear and Nonlinear Dispersion, Phys. Rev. Lett. 87 (2001), 1945-1948.

[14] Fokas A S and Fuchssteiner B, Symplectic Structures, their Bäcklund Transformations and Hereditary Symmetries, Physica D4 (1981), 47-66.

[15] Freeman N C and Davey A, On the Evolution of Packets of Long Surface Waves, Proc. Roy. Soc. A344 (1975), 427-433.

[16] Green G, On the Motion of Waves in a Variable Canal of Small Depth and Width, Camb. Trans. VI (1837) [Papers, p.255].

[17] Hasimoto H and Ono H, Nonlinear Modulation of Gravity Waves, J. Phys. Soc. Japan 33 (1972), 805-811.

[18] Hietarinta J, A Search for Bilinear Equations Passing Hirota's Three-Soliton Condition. I KdV-Type Bilinear Equations, J. Math. Phys. 28 (1987), 1732-1742.

[19] Johnson R S, On the Modulation of Water Waves on Shear Flows, Proc. Roy. Soc. A347 (1976), 537-546.

[20] Johnson R S, On the Modulation of Water Waves in the Neighbourhood of $k h \approx 1.363$, Proc. Roy. Soc. A357 (1977), 131-141.

[21] Johnson R S, On the Nonlinear Critical Layer below a Nonlinear Unsteady Surface Wave, J. Fluid Mech. 167 (1986), 327-351.

[22] Johnson R S, On Solutions of the Burns Condition (which Determines the Speed of Propagation of Linear Long Waves on a Shear Flow with or without a Critical Layer), Geophys. Astrophys. Fluid Dynamics 57 (1991), 115-133.

[23] Johnson R S, Solitary Wave, Soliton and Shelf Evolution over Variable Depth, J. Fluid Mech. 276 (1994), 125-138. 
[24] Johnson R S, A Two-Dimensional Boussinesq Equation for Water Waves and Some of Its Solutions, J. Fluid Mech. 323 (1996), 65-78.

[25] Johnson R S, Nonlinear Gravity Waves on the Surface of an Arbitrary Shear Flow with Variable Depth, in Nonlinear Instability Analysis, Editors: Debnath L and Choudhury S R, Comp. Mech. Publications, 1997, 221-243.

[26] Johnson R S, Camassa-Holm, Korteweg-de Vries and Related Models for Water Waves, J. Fluid Mech. 455 (2002), 63-82.

[27] Johnson R S, On Solutions of the Camassa-Holm Equation, Proc. R. Soc. Lond. A459 (2003), 1687-1708.

[28] Johnson R S, The Camassa-Holm Equation for Water Waves Moving over a Shear Flow, Fluid Dyn. Res. 33, Nr. 1-2 (2003), 97-111.

[29] Kadomtsev B P and Petviashvili V I, On the Stability of Solitary Waves in Weakly Dispersing Media, Sov. Phys. Dokl. 15 (1970), 539-541.

[30] Knickerbocker C J and Newell A C, Shelves and the Korteweg-de Vries Equation, J. Fluid Mech. 98 (1980), 803-818.

[31] Knickerbocker C J and Newell A C, Reflections from Solitary Waves in Channels of Decreasing Depth, J. Fluid Mech. 153 (1985), 1-16.

[32] Korteweg D J and de Vries G, On the Change of Form of Long Waves Advancing in a Rectangular Canal, and on a New Type of Long Stationary Waves, Phil. Mag. (5) 39 (1895), 422-443.

[33] Maslowe S A and Redekopp L G, Long Nonlinear Waves in Stratified Shear Flows, J. Fluid Mech. 101 (1980), 321-348.

[34] Matveev V B and Salle M A, Darboux Transformations and Solitons, Springer-Verlag, Berlin, 1991.

[35] Maxon S and Viecelli J, Cylindrical Solitons, Phys. Fluids 17 (1974), 1614-1616.

[36] Miles J W, Obliquely Interacting Solitary Waves, J. Fluid Mech. 79 (1977), 157-169.

[37] Miles J W, On the Korteweg-de Vries Equation for a Gradually Varying Channel, J. Fluid Mech. 91 (1979), 181-190.

[38] Rayleigh Lord [J W Strutt], On Waves, Phil. Mag. (5) 1 (1876), 257-279.

[39] Russell J S, Report on Waves, in Rep. 14th Meet. Brit. Assoc. Adv. Sci., York, John Murray, London, 1844, 311-390.

[40] Stokes G G, On the Theory of Oscillatory Waves, Trans. Camb. Phil. Soc. 8 (1847), 441455. 